\title{
El arte rupestre del sur de Mendoza en perspectiva biogeográfica: primeros resultados a escala regional
}

\author{
Agustín Acevedo, Danae Fiore, Hugo Tucker, Gustavo A. Neme y Adolfo F. Gil \\ Recibido 15 de noviembre 2019. Aceptado 27 de abril 2020
}

\begin{abstract}
RESUMEN
Este trabajo presenta los primeros resultados de un análisis regional del arte rupestre del sur de Mendoza, basado en el estudio de la distribución de 96 sitios en tres áreas biogeográficas, evaluadas según su productividad. La información obtenida permitió identificar un repertorio rupestre de diez clases de motivos, distribuidas en las tres áreas y generalmente combinadas entre sí en los sitios: ello sugiere la existencia de amplios procesos de comunicación visual al interior de la región estudiada. Sin embargo, también se han identificado procesos de índole más local en: a) la no-combinación de ciertas clases (e.g., cefalomorfasmascariformes vs. geométricos complejos rectilíneos), su producción con técnicas diferentes (e.g., grabado vs. pintura) y su emplazamiento diferencial (e.g., sin reparo vs. con reparo); b) las frecuencias diferenciales de sitios en algunos espacios de baja productividad, pero con recursos críticos -como La Payunia-, que sugieren posibles señalizaciones de dichos recursos en un contexto de intensificación durante el Holoceno reciente.
\end{abstract}

Palabras clave: Arte rupestre; Clases de motivos; Biogeografía; Productividad.

\begin{abstract}
SOUTHERN MENDOZA ROCK ART IN BIOGEOGRAPHICAL PERSPECTIVE: FIRST REGIONAL-SCALE RESULTS. This paper presents the initial results of a regional analysis of rock art in southern Mendoza based on the study of the distribution of 96 archaeological sites in three biogeographical areas and assessed according to their productivity. The information obtained helped us identify a repertoire of ten motif classes distributed throughout the three areas, though mostly concentrated at the sites. The distribution suggests the existence of widespread communication processes within the region. Other local processes have also been identified, including: a) the non-combination of certain motif classes (e.g., headshaped-maskshaped and complex rectilinear geometric motifs), the use of different production techniques, and the differential location of motifs on the rock face (e.g., sheltered or unsheltered); and b) the different frequency of sites in low productivity areas with critical resources - such as La Payunia - which suggests possible signaling of resources in a context of intensification during the recent Holocene.
\end{abstract}

Keywords: Rock art; Motif classes; Biogeography; Productivity.

Agustín Acevedo. Consejo Nacional de Investigaciones Científicas y Técnicas (CONICET). Asociación de Investigaciones Antropológicas (AIA). Bartolomé Mitre $11317^{\circ} \mathrm{G}$ (CP 1036), Ciudad Autónoma de Buenos Aires, Argentina. E-mail: agustinacevedo2009@gmail.com

Danae Fiore. CONICET. Universidad de Buenos Aires (UBA). AIA. Bartolomé Mitre $1|3| 7^{\circ}$ G (CP I036), Ciudad Autónoma de Buenos Aires, Argentina. E-mail: danae_fiore@yahoo.es

Hugo Tucker. Centro Regional de Investigación y Desarrollo Cultural-Malargüe (CRIDC). Museo Regional Malargüe (MRM), Av. San Martin esq. Pasaje La Orteguina (CP 56/3), Malargüe, Argentina. E-mail: tuckerhugo@gmail.com

Gustavo A. Neme. CONICET. Instituto de Evolución, Ecología Histórica y Ambiente (IDEVEA - CONICET y UTN FRSR). Av. Urquiza 350 (CP 5600), San Rafael, Argentina. E-mail: gneme@mendoza-conicet.gob.ar

Adolfo F. Gil. CONICET. Universidad Nacional de Cuyo (UNCuyo). IDEVEA - CONICET y UTN FRSR. Av. Urquiza 350 (CP 5600), San Rafael, Argentina. E-mail: agil@mendoza-conicet.gob.ar

Intersecciones en Antropología 21(2), julio-diciembre: 145-157. 2020. ISSN-e 1850-373X

https://doi.org/10.37176/iea.21.2.2020.494

Facultad de Ciencias Sociales - UNICEN - Argentina 


\section{INTRODUCCIÓN}

Desde hace más de una década, la arqueología del sur de Mendoza se ha focalizado en comprender la variabilidad del registro regional desde una perspectiva biogeográfica (Neme et al., 2005; Gil, 2006; Gil et al., 2006; Neme, 2007; Neme y Gil, 2008, 2012, entre otros). La adopción de este enfoque y su aplicación a escala regional propició el surgimiento de múltiples líneas de investigación (e.g., estudios líticos, arqueofaunísticos, arqueobotánicos, bioarqueológicos, cerámicos, isotópicos, etc.) y su integración en torno a objetivos comunes, vinculados con la comprensión de procesos de cambio cultural dentro de un contexto ambiental heterogéneo como lo es el sur de Mendoza (Gil et al., 2006; Neme y Gil, 2008, 2012, entre otros). Sin embargo, este crecimiento de la arqueología regional no incluyó a las investigaciones rupestres, lo que generó un desfasaje entre el grado de resolución fáctica sobre las mencionadas líneas de evidencia y la información disponible para el arte parietal, principalmente de índole cualitativa y estilística.

En función de lo expuesto, este trabajo se propone realizar un primer abordaje biogeográfico sobre el arte rupestre del sur de Mendoza. El objetivo principal consiste en evaluar la existencia de tendencias diferenciales en la producción de arte rupestre entre áreas biogeográficas que difieren en sus estructuras de recursos y productividad (Altoandina, Patagonia y Monte). Un segundo nivel de análisis implicará la evaluación de potenciales tendencias en La Payunia, un espacio de baja productividad que abarca sectores de Patagonia y Monte. Con este fin, se integran los sitios con arte del sur de Mendoza actualmente conocidos $(N=96)$-identificados a partir de la lectura de publicaciones antecedentes y en trabajos de campo del equipoy se analizan sus frecuencias, distribuciones y clases de motivos. Los resultados obtenidos permiten evaluar y discutir la existencia de relaciones entre la producción rupestre regional, la productividad ambiental y la dinámica de poblamiento humano del sur de Mendoza.

\section{ÁREA DE ESTUDIO}

La región bajo estudio comprende una superficie aproximada de $87.000 \mathrm{~km}^{2}$, limitada por el río Diamante al norte, el río Barrancas-Colorado al sur, la divisoria de aguas de la cordillera andina al oeste y el río Desaguadero-Salado al este (Figura 1) (Neme y Gil, 2008). Este territorio, de características generales semiáridas, puede definirse como un ambiente heterogéneo, atravesado por un gradiente altitudinal y pluvial este-oeste que configura tres áreas biogeográficas diferentes (Figura 1): a) Altoandina, que incluye las zonas de cordillera por encima de los ca. 2200 msnm; b) Patagónica, la cual incluye las zonas de piedemonte, valles intermontanos y el campo volcánico de La Payunia comprendidas entre los ca. 2200 y 1500 msnm; y c) Monte, extendida sobre las planicies orientales de la región y parte de La Payunia, con altitudes por debajo de los ca. 1500 msnm (Neme et al., 2005; Neme y Gil, 2008, 2012). La definición de estas áreas sigue la división de provincias fitogeográficas realizada por Cabrera

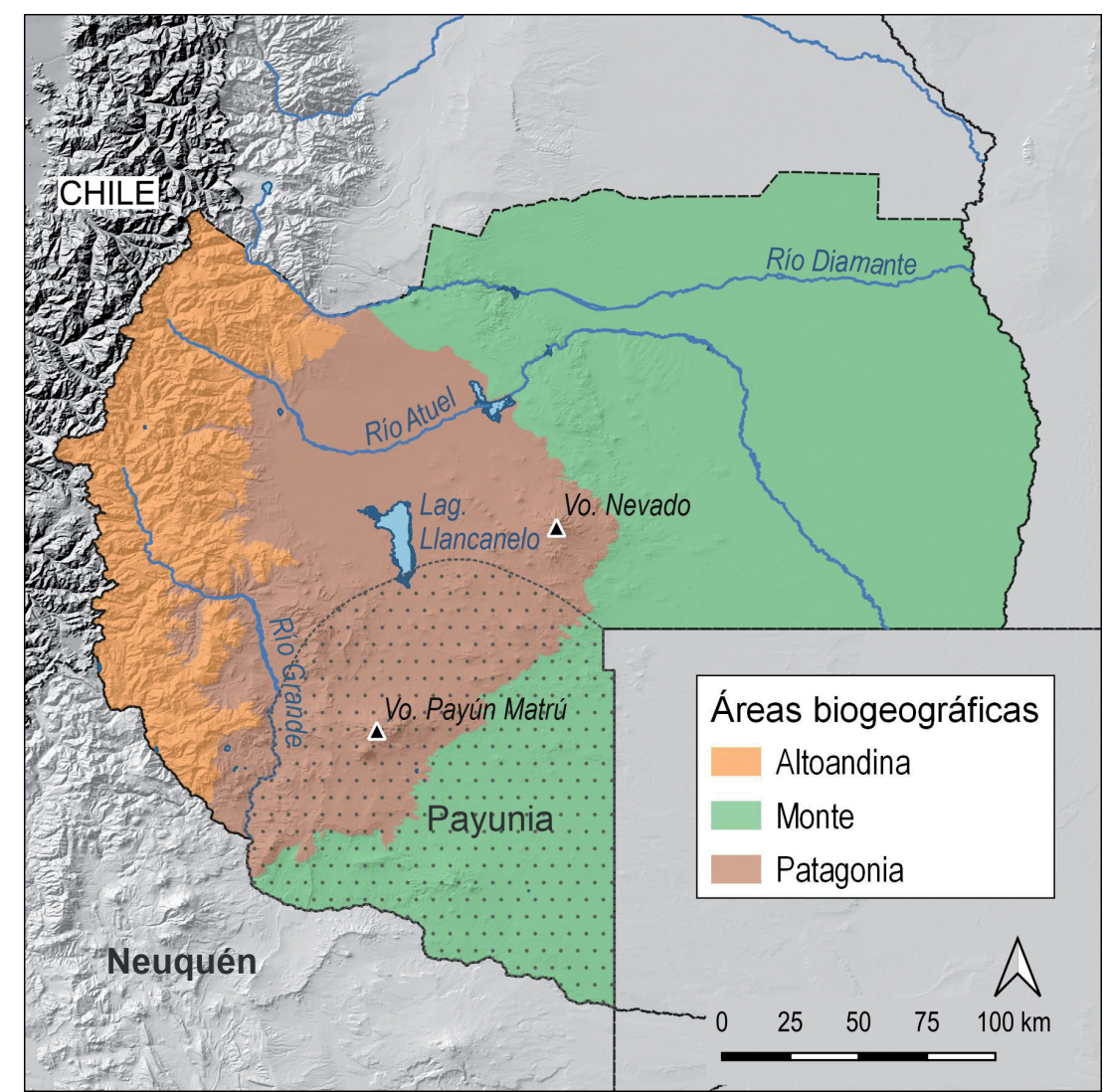

Figura 1. Región sur de Mendoza dividida en áreas biogeográficas. 
(1976) para el sur de Mendoza, las cuales guardan estrechas relaciones con las variaciones de los niveles altitudinales y el grado de continentalidad de los espacios regionales, por lo que reflejan los principales cambios botánicos, topográficos, geológicos, zoogeográficos y climáticos de la región (Neme y Gil, 2008, 2012).

Las diferencias existentes entre las distintas áreas biogeográficas -en términos de características geomorfológicas, climáticas y ecológicas- habrían generado distintos contextos y desafíos para el establecimiento de poblaciones humanas a lo largo del tiempo (Neme et al., 2005; Neme y Gil, 2008, 2012). El ordenamiento jerárquico de estas áreas, de acuerdo con un ranking de productividad sustentado en sus respectivas características ambientales estructurales, muestra que Patagonia -especialmente en los espacios del piedemonte andino- es el área más productiva, seguida por el Monte y luego Altoandina (Neme et al., 2005; Neme y Gil, 2008, 2012). Patagonia se caracteriza por ser el área con mayor productividad de guanacos en toda la región; allí el agua es más ubicua que en el Monte, y su diversidad vegetal, si bien es menor que en el Monte, es mayor que en el área Altoandina. A su vez, el Monte tiene el segundo lugar en este ranking básicamente por la alta productividad de las plantas y la homogeneidad de su distribución, lo cual genera una continuidad productiva en toda su extensión. Por el contrario, el área Altoandina, a pesar de las vegas y pastizales de altura con alta densidad de camélidos, posee una proporción muy importante de espacios totalmente improductivos como consecuencia de la presencia de fuertes pendientes, afloramientos rocosos y glaciares. Cada una de estas grandes áreas biogeográficas manifiesta una marcada heterogeneidad interna y esconde limitantes para la ocupación humana, como por ejemplo, los sectores de Monte y Patagonia correspondientes a La Payunia, donde la escasez de fuentes de agua superficial genera un fuerte descenso de la productividad.

\section{ANTECEDENTES DE INVESTIGACIÓN: ARQUEOLOGÍA REGIONAL Y ARTE RUPESTRE}

Las investigaciones desarrolladas durante los últimos 20 años en las tres áreas biogeográficas del sur de Mendoza han permitido generar una secuencia arqueológica que se inicia hacia finales del
Pleistoceno (ca. 11.000-10.000 años AP) y culmina en el Holoceno tardío final (ca. 2000-200 años AP) (Semper y Lagiglia, 1968; Lagiglia, 1977, 1980; Gil, 1997-1998; Neme y Gil, 2008, 2009, 2012). Dentro de esta secuencia, el área de Patagonia muestra una señal arqueológica más continua, intensa y estable a lo largo del Holoceno, mientras que las áreas de Monte y Altoandina tienen señales comparativamente más tardías y discontinuas (Neme y Gil, 2008, 2009, 2012). El principal modo de subsistencia registrado a lo largo del tiempo es la caza y recolección. Sin embargo, esto cambia en el Holoceno tardío final (ca. 2000-200 años AP), cuando los grupos ubicados al norte de los ríos Atuel y Diamante comenzaron a incorporar productos agrícolas a sus dietas (maíz, zapallo, poroto y quinoa), mientras que los situados al sur continuaron con un modo de subsistencia cazador-recolector (Lagiglia, 1980; Gil, 1997-1998). Actualmente se discute si la incorporación de estos cultígenos estuvo vinculada al desarrollo de un proceso de intensificación (cf. Neme, 2007; Neme y Gil, 2012; Otaola et al., 2015) y si generó un proceso de divergencia cultural que culminó con la adopción de la agricultura al norte del río Atuel (cf. Gil et al., 2010; Gil y Neme, 2013).

Las investigaciones antecedentes sobre el arte rupestre enmarcan cronológicamente su producción dentro de los últimos 2000 años de ocupación humana de la región, a partir de la utilización de indicadores estilísticos (cf. Lagiglia, 1977, 2003, 2004; Schobinger, 1978, 2002; Gradin, 19971998). En términos generales, se trata de publicaciones focalizadas en la descripción de sitios y motivos rupestres, en función de objetivos vinculados con la definición de estilos, su adscripción cultural y la generación de cronologías relativas. Estas se desarrollaron en dos sectores de la región: el sector norte, representado por los sitios comprendidos entre los valles de los ríos Atuel y Diamante; y el sector sur, representado por los sitios ubicados al sur del río Atuel, hasta el río Barrancas-Colorado (cf. Lagiglia, 1977; Schobinger, 1978, 2002; Gradin, 1997-1998).

En el sector norte se identificaron tres zonas con sitios rupestres: a) el valle del río Atuel, que incluye la localidad arqueológica Rincón del Atuel, en el curso medio del río, donde se registraron seis sitios con arte (Lagiglia, 1956a, 1956b, 1977, 1997, 2003, 2004); b) el valle del río Diamante, donde se registraron dos sitios con arte; y c) Sierra Pintada, donde se registraron un total de cuatro sitios con arte (Rusconi, 1962; Lagiglia, 1977). 
Dentro de la localidad Rincón del Atuel, Lagiglia distinguió dos grupos de pinturas diferentes. Por un lado, el grupo de pinturas del Estilo tipo zigzagueado, que el autor vinculó con la decoración cerámica Condorhuasi y atribuyó a grupos agricultores incipientes de la cultura Atuel II (ca. 2300-1900 años AP) (Lagiglia, 1956a, 1956b, 1962-1968, 1977). Por otro lado, el grupo de pinturas de Gruta del Indio, compuesto por motivos geométricos, tridígitos y otros, atribuido por el autor a grupos cazadoresrecolectores araucanizados de la cultura Atuel I (ca. 500-200 años AP) (Lagiglia, 1956a, 1956b, 19621968, 1977, 2004).

En el resto del sector, los trabajos fueron generalmente menos exhaustivos. En el valle del río Diamante se describieron dos sitios con arte: Arroyo del Tigre, con pinturas geométricas, y Reparo Patas de Pumas, con pisadas de animales pictograbadas (Rusconi, 1962; Lagiglia, 1977). En la porción de Sierra Pintada, ubicada entre los ríos Diamante y Atuel, se describieron tres sitios con pinturas geométricas complejas: Cueva del Cacique, Cueva de las Guardas y Gruta Las Tinajas, esta última atribuida a grupos agricultores Huarpes del horizonte cultural Tinajas I (ca. 500-200 años AP) (Rusconi, 1962; Lagiglia, 1977, 2003). También en Sierra Pintada, pero al sur del Atuel, en el sitio Ponontrehue, se describió la presencia de grabados mascariformes adscriptos al Estilo de rostros, vinculado a grupos cazadores-recolectores (Lagiglia, 1997).

Al sur del río Atuel se realizaron trabajos con perspectivas más regionales que destacaron la variabilidad estilística observada en el sector. Schobinger $(1978,2002)$ registró 23 sitios con arte. En el caso de los grabados, distinguió cuatro modalidades vinculadas a distintas regiones vecinas (e.g., Cuyo, NOA, Patagonia y el río Maule chileno): a) tendencia curvilínea irregular; b) cabezas y figuras antropomorfas mascariformes; c) tridígitos; y d) geometrismo de Valle Hermoso (Schobinger, 1978, 2002). En el caso de las pinturas, estas se habrían producido con posterioridad a los grabados y representarían una penetración del Estilo de grecas patagónico entre ca. 1400 y 500 años AP (Schobinger, 1978, 2002). Por su parte, apoyándose en los relevamientos realizados por Schobinger (1978), Gradin (19971998) diferenció tres modalidades estilísticas en el departamento Malargüe: a) grabados de procedencia andina (mascariformes), atribuidos a cazadores y horticultores-alfareros de ca. 1300 años AP; b) pinturas geométricas complejas (grecas), introducidas por grupos cazadores-recolectores patagónicos en ca. 1000 años AP; y c) geométrico simple, vinculado con las sierras de San Luis entre ca. 800-500 años AP. Cabe destacar que estos autores no realizaron trabajos de excavación en la región, por lo que recurrieron a asociaciones de índole estilística y les asignaron cronologías relativas mediante información cronológica de regiones vecinas.

Con posterioridad a estos trabajos, las investigaciones sobre arte rupestre se enfocaron hacia preguntas de investigación más específicas. Así, desde una perspectiva estética de las artes plásticas, Hart (2015) realizó un análisis de recursos compositivos utilizados en la configuración de motivos antropomorfos y geométricos en diferentes sitios de la región; y Tucker y colaboradores (2011) evaluaron la representación de fenómenos astronómicos en la disposición espacial de morteros asociados a grabados rupestres del departamento Malargüe.

En síntesis, los antecedentes sobre el arte rupestre del sur de Mendoza consisten principalmente en investigaciones histórico-culturales, arqueoastronómicas y artísticas, con bajos niveles de integración de la información rupestre a escala regional y desvinculadas de la discusión biogeográfica actual. Este desfasaje existente entre la arqueología regional y la arqueología del arte rupestre del sur de Mendoza es el que se pretende comenzar a corregir a partir del presente trabajo.

\section{MARCO TEÓRICO Y METODOLOGÍA: ARTE Y BIOGEOGRAFÍA EN LAS OCUPACIONES HUMANAS DEL ESPACIO}

Actualmente, parte de las investigaciones arqueológicas en el sur de Mendoza se enmarcan dentro de una perspectiva biogeográfica y ecológica evolutiva, que analiza el proceso de poblamiento humano y sus señales arqueológicas en relación con las transformaciones geomorfológicas y ecológicas de los espacios ocupados a lo largo del tiempo (Veth, 1989; Borrero, 1994-1995; Neme y Gil, 2008, 2012). Estas investigaciones parten de una premisa según la cual las decisiones humanas sobre la selección de los espacios a ser habitados están influenciadas por la productividad diferencial existente entre las distintas áreas biogeográficas de la región (Neme y Gil, 2008, 2012). A su vez, estas decisiones y prácticas se vinculan con otros factores, tales como la tecnología disponible para explotar diversos recursos, la densidad poblacional y la organización socioeconómica de los grupos 
que exploran, colonizan y ocupan una región (sensu Borrero, 1994-1995).

Dentro de estas decisiones y prácticas, la producción de arte rupestre involucra operaciones de selección de emplazamientos vinculadas a los rangos de acción y circuitos de movilidad de los grupos productores $y$, por lo tanto, a sus estrategias de subsistencia y a la estructura de recursos explotados (Conkey, 1984; Aschero, 1997; Martel, 2006, entre otros). Esto, sumado a la capacidad del arte para transmitir información a nivel intra e intergrupal, lo convierte en una herramienta activa en la organización de los espacios asociados a las prácticas de subsistencia y movilidad de los grupos humanos (Aschero, 1997; Hernández Llosas, 1997; Martel, 2006; Carden, 2009; Scheinsohn, 2011; Romero y Re, 2014). Debido a su naturaleza inmóvil, el arte rupestre es uno de los materiales de mayor resolución espacial dentro del registro arqueológico, lo cual lo convierte en un indicador relevante para la evaluación de procesos de producción plástica y comunicación visual que operan sobre soportes fijos en la selección/habitación/marcación de espacios dentro de contextos biogeográficos específicos, pero que a su vez pueden trascender estos contextos y operar también a escala macrorregional.

En función de lo expuesto, este trabajo retoma la perspectiva biogeográfica arriba mencionada asumiendo dos premisas: a) que la distribución espacial de los sitios con arte no es azarosa (Aschero, 1997; Hernández Llosas, 1997; Martel, 2006, entre otros); b) que la productividad de los espacios de cada área biogeográfica y las prácticas socioeconómicas de los grupos productores allí desarrolladas habrían sido factores relevantes que influyeron sobre dicha distribución.

Para efectuar el registro y análisis de datos, se aplicó una metodología orientada hacia la sistematización de información básica sobre el arte rupestre de la región y su caracterización tecnomorfológica a escala de sitio. El registro de datos incluyó información sobre sitios rupestres publicada en trabajos antecedentes y generada por el equipo a lo largo de 20 años de investigaciones en el sur de Mendoza, relevando seis variables: nombre del sitio, coordenadas, tipo de morfología del soporte rocoso (e.g., alero, cueva, paredón), materia prima lítica del soporte, clases de motivos y técnicas generales de ejecución (grabado y pintura).

La información registrada se volcó posteriormente en una base de datos Excel y en una base de datos espaciales -en forma de coberturas vectoriales- generada con Sistemas de Información Geográfica (SIG). La base de datos Excel se utilizó inicialmente para cuantificar los resultados generados por investigaciones antecedentes $y$, posteriormente, para la realización de análisis estadísticos univariados y bivariados orientados hacia la búsqueda de tendencias generales respecto de las frecuencias y distribuciones de sitios con arte, técnicas y clases de motivos entre las diferentes áreas biogeográficas de la región. Para evaluar las similitudes entre áreas biogeográficas en términos de repertorios y frecuencias de clases de motivos, se realizaron análisis de agrupamiento jerárquico con PAST (versión 3.25). El SIG se utilizó principalmente para seleccionar las variables relevantes a ser incluidas en los análisis estadísticos univariados y bivariados, explotando su capacidad para integrar información espacial y generar resúmenes visuales (Wheatley y Gillings, 2002).

Las clases de motivos fueron definidas agrupando tipos de motivos vinculados entre sí por afinidades formales (utilizando criterios tales como si el motivo está compuesto por líneas rectas, curvas, figuras perimetrales, figuras de cuerpo lleno, etc.) y temáticas (utilizando criterios relativos a la identificación del referente representado, cuando este es reconocible, tales como mascariforme-cefalomorfo o pisadas). La elección de esta categoría amplia para el análisis formal de las imágenes (por encima de categorías como grupo o tipo de motivo) respondió a la necesidad de abarcar motivos descriptos en la bibliografía con diferentes grados de detalle. Estas clasificaciones, aun siendo actuales y occidentales, sirven como herramienta para el análisis sistemático de las producciones visuales y la búsqueda de tendencias generales en el uso de las formas (Hernández Llosas, 1997).

\section{RESULTADOS}

\section{Caracterización de los emplazamientos rupestres de la región}

El procesamiento de la información registrada permitió contabilizar un total de 96 sitios con arte en el sur de Mendoza, de los cuales 90 pudieron ser ubicados a escala de áreas biogeográficas (Altoandina, Patagónica y Monte) y 87 pudieron ser georreferenciados (53 en forma precisa y 34 en forma aproximada) (Tabla 1; Figura 2A). El análisis de la distribución espacial de sitios por áreas 
biogeográficas muestra que 42 sitios $(46,7 \%)$ se localizan en Patagonia, 35 (38,9\%) en Monte y 13 $(14,4 \%)$ en Altoandina (Tabla 1; Figura 2B). Estos resultados sugieren la existencia de un vínculo entre la productividad de las áreas biogeográficas y la frecuencia de sitios con arte rupestre'. Sin embargo, al refinar el análisis, un resultado llamativo es que la mitad de los sitios con arte de Patagonia $(\mathrm{n}=$ $23 ; 54,8 \%$ ) y una cuarta parte de los sitios con arte del Monte $(n=9 ; 25,7 \%)$ se ubican en sectores correspondientes a La Payunia, uno de los espacios menos productivos de la región.

De los 96 sitios con arte registrados, $74(77,1 \%)$ poseen información sobre el tipo de morfología del

\begin{tabular}{|l|c|c|c|c|}
\hline $\begin{array}{l}\text { Área } \\
\text { biogeográfica }\end{array}$ & $\begin{array}{c}\mathbf{N} \\
\text { sitios }\end{array}$ & $\begin{array}{c}\mathbf{\%} \\
\text { Sitios }\end{array}$ & $\begin{array}{c}\mathbf{K m}^{\mathbf{2}} \\
(\mathbf{c a} .)\end{array}$ & $\begin{array}{c}\mathbf{N} \\
\text { sitios } \mathbf{k m}^{2}\end{array}$ \\
\hline Altoandina & 13 & 14,4 & 13.000 & 0,00100 \\
\hline Patagónica & 42 & 46,7 & 25.000 & 0,00168 \\
\hline Monte & 35 & 38,9 & 49.000 & 0,00071 \\
\hline $\begin{array}{l}\text { Total Áreas } \\
\text { biogeográficas }\end{array}$ & $\mathbf{9 0}$ & $\mathbf{1 0 0}$ & $\mathbf{8 7 . 0 0 0}$ & $\mathbf{0 , 0 0 1 0 3}$ \\
\hline
\end{tabular}

Tabla 1. Frecuencia de sitios con arte por área biogeográfica.
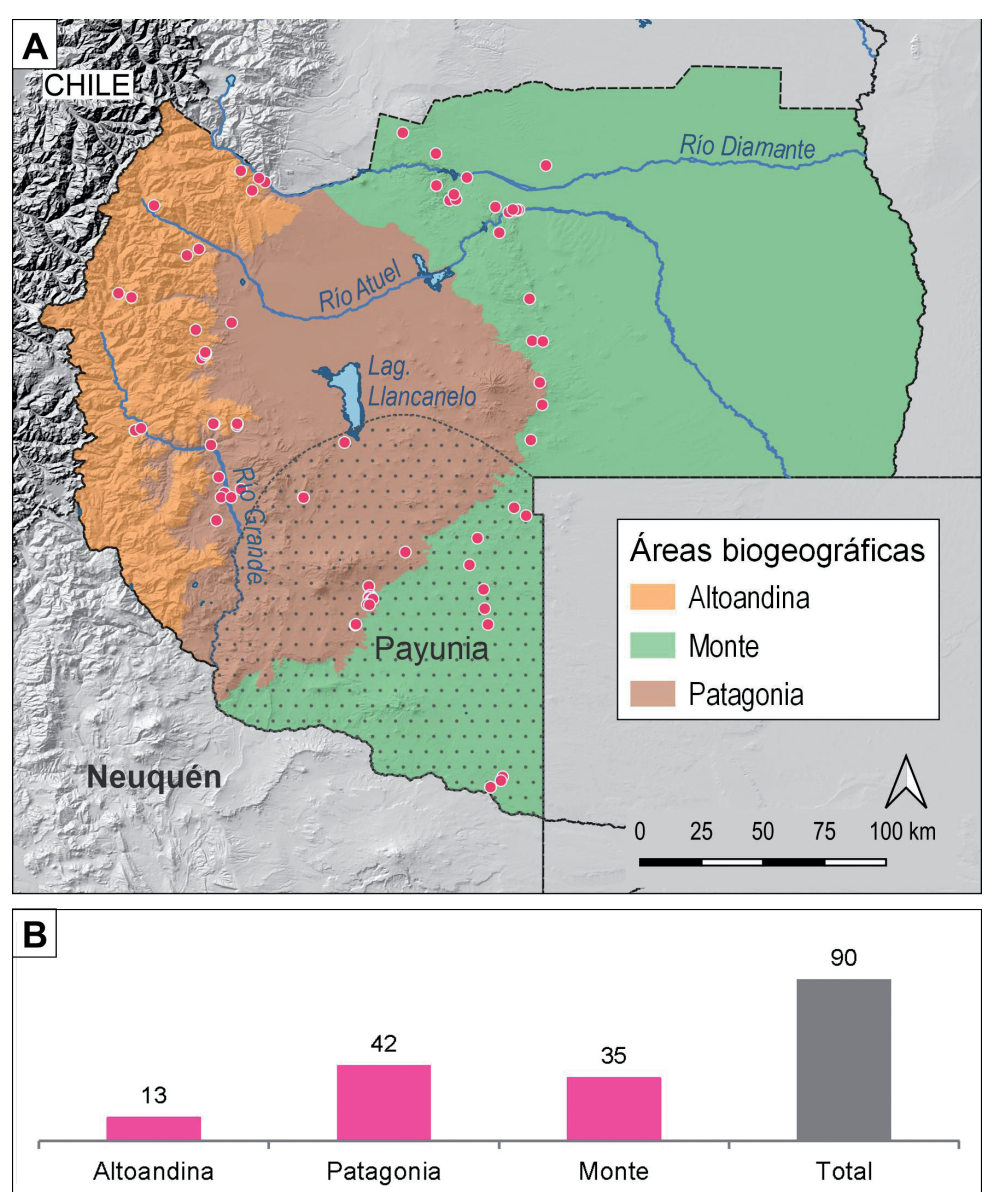

Figura 2. A) Sitios con arte rupestre del sur de Mendoza; B) Frecuencia de sitios con arte por área biogeográfica. soporte en que se encuentran emplazados (e.g., cueva, alero, etc.) y 57 de ellos ( $77 \%$ de 74$)$, sobre su materia prima rocosa (e.g., basalto, ignimbritas). Sin embargo, los criterios y términos utilizados en la bibliografía para caracterizar los sitios no siempre están explicitados ni son sistemáticos, lo cual genera ambigüedades al momento del registro de las variables y su posterior análisis ${ }^{2}$. Una evaluación conservadora de los tipos de morfologías y materias primas rocosas de los soportes utilizados indica, por un lado, la utilización de afloramientos rocosos de ignimbrita $(n=35 ; 61,4 \%)$ y basalto $(n=$ $22 ; 38,6 \%)$; por otro lado, una mayor utilización de emplazamientos no reparados (e.g., paredones y bloques: $n=42 ; 56,7 \%$ ), por sobre los reparados (e.g., aleros y cuevas: $\mathrm{n}=32 ; 43,3 \%$ ). Esta última tendencia difiere entre las áreas biogeográficas; así, mientras que en Monte y Altoandina predominan los emplazamientos reparados por sobre los no reparados $(\mathrm{n}=16$ vs. $\mathrm{n}=7$ y $\mathrm{n}=6$ vs. $\mathrm{n}=2$, respectivamente), en Patagonia se produce lo contrario (reparados: $\mathrm{n}=10$; no reparados: $\mathrm{n}=31$ ).

\section{Caracterización técnica y morfológica de la producción rupestre de la región}

La totalidad de los sitios con arte registrados $(\mathrm{N}=96)$ posee información relativa a las técnicas generales de producción de motivos rupestres. Estos datos muestran igual cantidad de sitios con pinturas y sitios con grabados ( $n=46$, respectivamente) y pocos casos de sitios con ambas técnicas de ejecución $(n=4)$ (Tabla 2).

A escala de áreas biogeográficas, las tres manifiestan tendencias técnicas diferentes (Tabla 2): a) en el área de Monte, los sitios pintados predominan sobre los grabados $(68,6 \%$ vs. $22,9 \%$; b) en el área Patagónica, los sitios grabados predominan sobre los pintados (69 \% vs. 31\%); y c) en el área Altoandina, ambas técnicas presentan frecuencias similares (pintura: $46,2 \%$ vs. grabado: $53,8 \%$ ).

De todos los sitios registrados $(\mathrm{N}=$ 96), $61(63,5 \%)$ poseen información relativa a la morfología de los motivos rupestres. Esta información fue 


\begin{tabular}{|l|c|c|c|c|c|c|c|c|}
\hline \multirow{2}{*}{$\begin{array}{c}\text { Áreas } \\
\text { biogeográficas }\end{array}$} & \multicolumn{2}{|c|}{ Pintura } & \multicolumn{2}{c|}{ Grabado } & \multicolumn{2}{c|}{$\begin{array}{c}\text { Grab. y } \\
\text { Pint. }\end{array}$} & \multicolumn{2}{c|}{ Total } \\
\cline { 2 - 10 } & $\boldsymbol{n}$ & $\boldsymbol{\%}$ & $\boldsymbol{n}$ & $\boldsymbol{\%}$ & $\boldsymbol{n}$ & $\boldsymbol{\%}$ & $\boldsymbol{n}$ & $\boldsymbol{\%}$ \\
\hline Altoandina & 6 & 46,2 & 7 & 53,8 & - & - & 13 & 100 \\
\hline Monte & 8 & 22,9 & 24 & 68,6 & 3 & 8,6 & 35 & 100 \\
\hline Patagónica & 29 & 69 & 13 & 31 & - & - & 42 & 100 \\
\hline No ubicado & 3 & 50 & 2 & 33,3 & 1 & 16,7 & 6 & 100 \\
\hline Total & $\mathbf{4 6}$ & $\mathbf{4 7 , 9}$ & $\mathbf{4 6}$ & $\mathbf{4 7 , 9}$ & $\mathbf{4}$ & $\mathbf{4 , 2}$ & $\mathbf{9 6}$ & $\mathbf{1 0 0}$ \\
\hline
\end{tabular}

Tabla 2. Frecuencia de sitios grabados, pintados y mixtos por área biogeográfica.

agrupada dentro de 10 clases de motivos: geométricos rectilíneos $(n=41)$, geométricos complejos rectilíneos $(\mathrm{n}=34)$, geométricos curvilíneos $(\mathrm{n}=$ $24)$, pisadas $(n=26)$, figuras cefalomorfas-mascariformes $(n=18)$, geométricos puntiformes $(n=15)$, figuras antropomorfas $(n=10)$, manos $(n=3)$, figuras zoomorfas $(n=2)$ y motivos históricos/grafitis $(\mathrm{n}=3)$ (Figura 3A).

Los resultados muestran que: a) la mayoría de las clases de motivos $(n=6)$ se encuentran presentes en las tres áreas (con excepción de las figuras cefalomorfas-mascariformes, ausentes en el área Altoandina; las figuras zoomorfas, ausentes en Patagonia; los motivos históricos, presentes solo en Monte; y las manos, presentes solo en Patagonia); b) las clases más frecuentes son las mismas en las tres áreas (geométricos rectilíneos, geométricos curvilíneos y geométricos complejos rectilíneos); c) se registran excepciones en los casos de las pisadas (más frecuentes en Patagonia y Monte que en el área

\begin{tabular}{|l|c|c|c|c|}
\hline $\begin{array}{l}\text { Clases de motivos } \\
\text { (CM) }\end{array}$ & $\begin{array}{c}\text { Altoan- } \\
\text { dina }\end{array}$ & $\begin{array}{c}\text { Mon- } \\
\text { te }\end{array}$ & $\begin{array}{c}\text { Patagó- } \\
\text { nica }\end{array}$ & $\begin{array}{c}\text { N de } \\
\text { sitios }\end{array}$ \\
\hline Antropomorfos & 2 & 2 & 6 & $\mathbf{1 0}$ \\
\hline $\begin{array}{l}\text { Cefalomorfas - } \\
\text { mascariformes }\end{array}$ & - & 4 & 14 & $\mathbf{1 8}$ \\
\hline Zoomorfos & 1 & 1 & - & $\mathbf{2}$ \\
\hline Geom. Curvilíneos & 3 & 10 & 11 & $\mathbf{2 4}$ \\
\hline $\begin{array}{l}\text { Geom. Comp. } \\
\text { Rectilíneos }\end{array}$ & 3 & 15 & 16 & $\mathbf{3 4}$ \\
\hline Geom. Puntiformes & 1 & 6 & 8 & $\mathbf{1 5}$ \\
\hline Geom. Rectilíneos & 3 & 15 & 23 & $\mathbf{4 1}$ \\
\hline Manos & - & - & 3 & $\mathbf{3}$ \\
\hline Pisadas & 1 & 10 & 15 & $\mathbf{2 6}$ \\
\hline Motivos históricos & - & 3 & - & $\mathbf{3}$ \\
\hline N CM & $\mathbf{7}$ & $\mathbf{9}$ & $\mathbf{8}$ & $\mathbf{1 0}$ \\
\hline N Sitios c/ CM & $\mathbf{4}$ & $\mathbf{2 3}$ & $\mathbf{3 4}$ & $\mathbf{6 1}$ \\
\hline
\end{tabular}

Tabla 3. Frecuencia de clases de motivos por sitio a escala de área biogeográfica.
Altoandina) y en las figuras cefalomorfasmascariformes (más frecuentes en Patagonia que en el resto de las áreas) (Tabla 3; Figura $4 \mathrm{~A})$. Esto pone en evidencia que la mayoría de las clases de motivos registradas circularon entre las tres áreas biogeográficas de la región (Figura 3B y Figura 4A). Un análisis de agrupamiento jerárquico permite observar que -en términos de repertorios y frecuencias de clases-, las áreas de Monte y Patagonia registran mayores similitudes entre sí que con el área Altoandina. En forma complementaria, un segundo nivel de análisis muestra que los sitios correspondientes al sector de La Payunia, tanto en Monte como en Patagonia, exhiben mayores similitudes con sitios de otros sectores de Patagonia (e.g., Valles intermontanos y Piedemonte) y menores similitudes con otros sitios del área de Monte, lo cual permite sugerir que la heterogeneidad interna de cada una de las áreas biogeográficas se expresa en afinidades entre sectores específicos dentro de ellas, visibles en el registro arqueológico artístico (Figura 4B).

El análisis de las técnicas generales de producción artística muestra que la mayoría de las clases $(n=6)$ fueron realizadas tanto con técnicas de grabado como de pintura. Solo en tres de las clases se observan relaciones significativas entre técnica y morfología: las figuras cefalomorfas-mascariformes exclusivamente grabadas $(100 \%)$, las pisadas principalmente grabadas $(73,1 \%)$ y los geométricos complejos rectilíneos mayoritariamente pintados $(73,5 \%)$. Las clases restantes, o bien exhiben paridad de técnicas (e.g., figuras antropomorfas, geométricos curvilíneos, geométricos rectilíneos y geométricos puntiformes), o bien poseen frecuencias muy bajas como para extraer tendencias significativas (e.g., figuras zoomorfas, manos y motivos históricos).

Por último, el análisis de las combinaciones de clases de motivos por sitio muestra que todas las clases se combinan con otras, es decir, ninguna de ellas aparece aislada (Figura 5). Las combinaciones más frecuentes son aquellas que reúnen a las clases más frecuentes (geométricos rectilíneos, geométricos curvilíneos, geométricos complejos rectilíneos, pisadas, figuras cefalomorfas-mascariformes) (Figura 5), lo cual es estadísticamente esperable de una combinación azarosa y por lo tanto, no permite realizar inferencias significativas. 


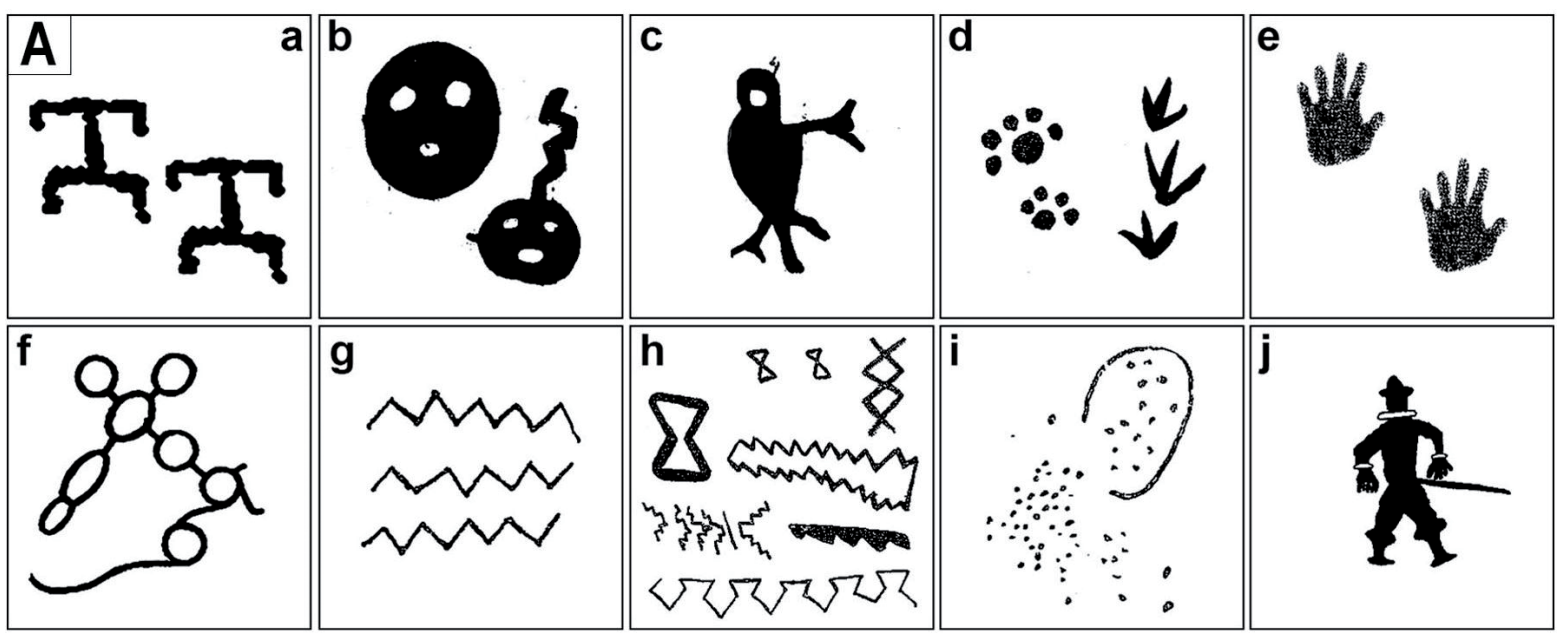

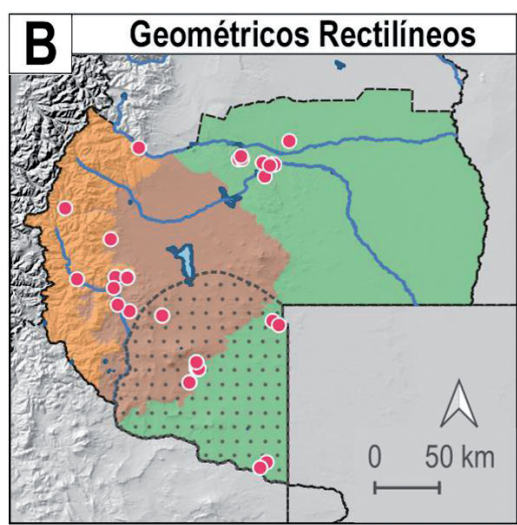

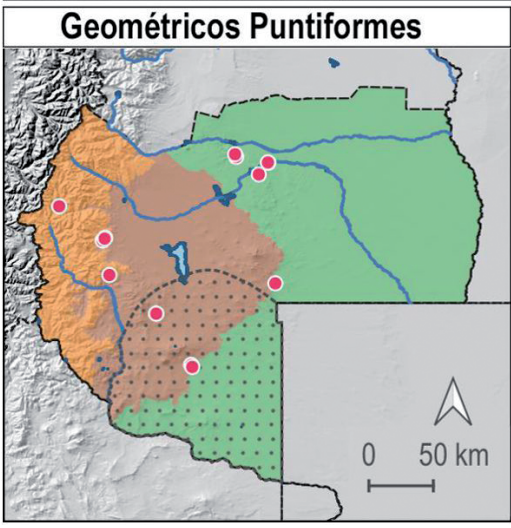

Figura 3. A) Clases de motivos del sur de Mendoza: a) figuras antropomorfas; b) figuras cefalomorfas-mascariformes; c) figuras zoomorfas; d) pisadas; e) manos; f) geométricos curvilíneos; g) geométricos rectilíneos; h) geométricos complejos rectilíneos; i) geométricos puntiformes; j) motivos históricos/grafitis. B) Distribución espacial de las clases de motivos más frecuentes de la región.

Sin embargo, dentro de este escenario, resulta llamativo el comportamiento de dos clases de motivos: geométricos complejos rectilíneos y figuras cefalomorfas-mascariformes que, a pesar de ser dos clases frecuentes dentro de la muestra y de ubicarse -en algunas ocasiones- en sitios muy próximos (Figura 3B), se combinan muy poco entre sí (Figura 5). Una evaluación más detallada de estas dos clases de motivos muestra fuertes contrastes entre ellas: a) las figuras cefalomorfas-mascariformes son exclusivamente grabadas $(n=18$;
$100 \%$ ), mientras que los geométricos complejos rectilíneos son mayoritariamente pintados $(n=25$; $73,5 \%)$; b) las figuras cefalomorfas-mascariformes tienden a ubicarse sobre soportes no-reparados $(\mathrm{n}=$ $16 ; 88,9 \%$ ) y los geométricos complejos rectilíneos sobre soportes reparados ( $\mathrm{n}=22 ; 64,7 \%)$; c) las figuras cefalomorfas-mascariformes se distribuyen principalmente por Patagonia $(n=14 ; 77,7 \%)$ y dentro de Monte, en el sector correspondiente a La Payunia ( $n=4 ; 22,2 \%)$, siempre al sur del río Atuel, mientras que los geométricos complejos rectilíneos 

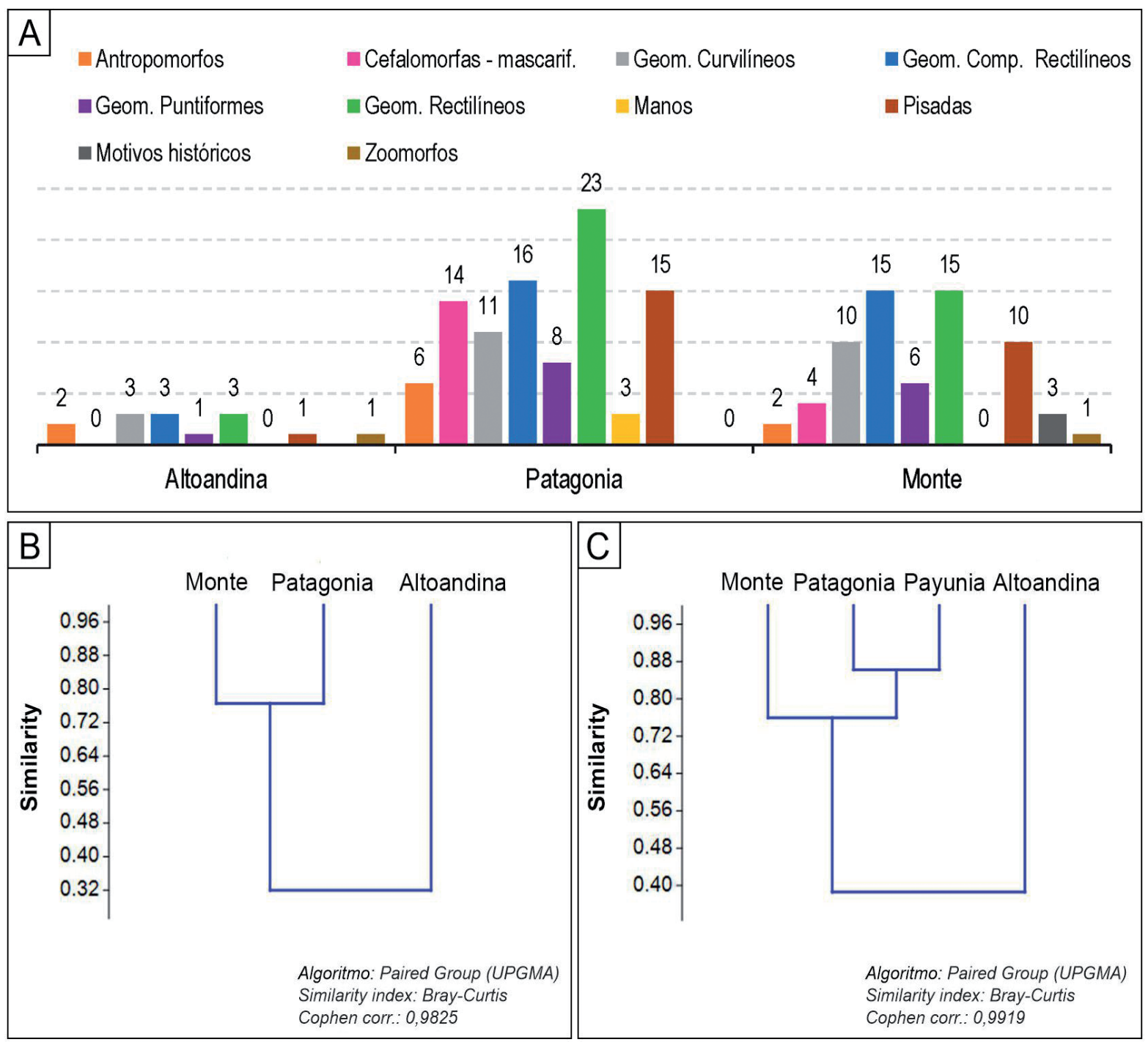

Figura 4. A) Frecuencia de clases de motivos por áreas biogeográficas; B) Análisis de agrupamiento jerárquico entre áreas biogeográficas por clases de motivos; C) Análisis de agrupamiento jerárquico entre áreas biogeográficas y La Payunia por clases de motivos.

lo hacen de manera más homogénea entre Monte $(\mathrm{n}=15 ; 44,1 \%)$ y Patagonia $(\mathrm{n}=16 ; 47,0 \%)$, en menor medida, el área Altoandina $(\mathrm{n}=3 ; 8,8 \%)$, incluyendo el norte del río Atuel. Estas divergencias se vuelven más pronunciadas cuando los análisis se focalizan sobre los motivos geométricos complejos rectilíneos pintados $(n=25)$, los cuales se ubican principalmente sobre soportes reparados $(n=21$; $84 \%)$, localizados frecuentemente en el área de Monte $(n=12 ; 48 \%)$, tanto al norte como al sur del río Atuel, y nunca se combinan con las figuras cefalomorfas-mascariformes. Estos resultados ponen en evidencia la existencia de pautas de producción diferentes entre ambas clases de motivos y la necesidad de comenzar a indagar sobre las causas subyacentes a estos patrones diferenciales.

\section{DISCUSIÓN Y CONCLUSIONES: DE COMUNICACIÓN REGIONAL HOMOGÉNEA $Y$ TENDENCIAS PUNTUADAS}

De acuerdo con la información arqueológica preexistente, las tres áreas biogeográficas del sur de Mendoza muestran ocupaciones desde momentos tempranos, pero mientras que Patagonia tiene una señal más intensa y estable a lo largo del Holoceno, Monte y Altoandina muestran señales más bien tardías y discontinuas (Semper y Lagiglia, 1968; Dieguez y Neme, 2003; Neme et al., 2005, 2011; Neme y Gil, 2008, 2012). A su vez, los sectores menos productivos, como el campo volcánico de La Payunia, muestran solamente ocupaciones correspondientes a momentos finales del Holoceno tardío (Gil, 2006; Neme y Gil 2008, 2012). En este 


\begin{tabular}{|c|c|c|c|c|c|c|c|c|c|c|c|}
\hline & 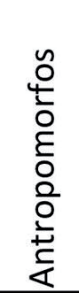 & 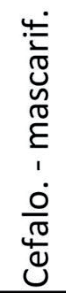 & 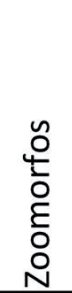 & 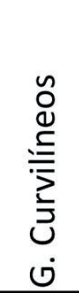 & 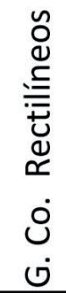 & 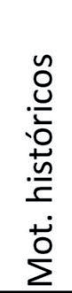 & 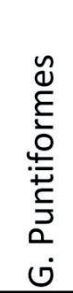 & 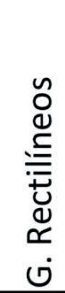 & $\begin{array}{l}\tilde{0} \\
\stackrel{0}{\pi} \\
\sum\end{array}$ & 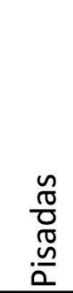 & 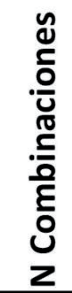 \\
\hline Antropomorfos & & & & & & & & & & & 8 \\
\hline Cefalo. - mascarif. & 2 & & & & & & & & & & 8 \\
\hline Zoomorfos & 1 & 1 & & & & & & & & & 8 \\
\hline G. Curvilíneos & 7 & 5 & 2 & 1 & & & & & & & 8 \\
\hline G. Co. Rectilíneos & 7 & 2 & 1 & 18 & & & & & & & 8 \\
\hline Mot. históricos & 1 & 0 & 0 & 2 & 3 & & & & & & 6 \\
\hline G. Puntiformes & 1 & 5 & 1 & 7 & 9 & 1 & & & & & 9 \\
\hline G. Rectilíneos & 9 & 10 & 1 & 20 & 23 & 3 & 11 & & & & 8 \\
\hline Manos & 0 & 3 & 0 & 0 & 0 & 0 & 3 & & & & 3 \\
\hline Pisadas & 6 & 11 & 2 & 15 & 13 & 1 & 11 & 19 & 3 & & 9 \\
\hline N Clases de Motivos & 10 & 18 & 2 & 24 & 34 & 3 & 15 & 41 & 3 & 26 & \\
\hline
\end{tabular}

Figura 5. Combinaciones de clases de motivos por sitio.

marco, el análisis de la frecuencia de sitios con arte rupestre por áreas biogeográficas revela una correlación entre cantidad de sitios y productividad. Así, el área Patagónica -de mayor productividad- posee la mayor cantidad de sitios con arte $(n=42)$, seguida por el área de Monte $(n=35)$, que la secunda en términos de productividad $y$, en tercer lugar, el área Altoandina ( $n=13)$, que es la de menor productividad. Esta tendencia es consistente con la correlación entre la productividad de las áreas biogeográficas y la intensidad de ocupación y reocupación humana verificadas en numerosos tipos de evidencia tales como las frecuencias de fechados y la densidad de materiales líticos y cerámicos, que reflejan una covariación de tasas de descarte respecto de la productividad de cada área (Neme et al., 2005; Neme y Gil, 2008, 2012).

Si bien esta correlación a escala regional responde de manera bastante lineal a las expectativas derivadas a partir de la productividad de cada área sobre la frecuencia de sitios con arte, resultan llamativos los resultados de nuestro segundo nivel de análisis, que demuestran que, en las áreas de Monte y Patagonia, los sectores correspondientes a La Payunia presentan una gran cantidad de sitios con arte a pesar de ser espacios poco productivos. Si se toman como válidas las dataciones relativas del arte asignadas por investigadores antecedentes -que lo sitúan dentro de los últimos 2000 años de ocupación-, entonces la elevada frecuencia de sitios rupestres en un sector poco productivo como La Payunia podría estar vinculada con un incremento en la señalización y/o demarcación de recursos críticos que se encontraran dentro de este sector, en un contexto de intensificación y competencia por los recursos ya discutido en trabajos previos (cf. Neme, 2007; Neme y Gil, 2012; Otaola et al., 2015). En tal sentido, Gil (2006) ya destacó la relación existente entre la distribución espacial de los sitios con arte en La Payunia y su cercanía con recursos críticos como fuentes de agua. Proponemos entonces como interpretación preliminar de esta tendencia que la utilización frecuente de soportes no reparados en este sector ( $n=15 ; 68,9 \%$ ) y de técnicas de producción comparativamente resistentes al deterioro -como el grabado- podrían representar elecciones vinculadas con el favorecimiento de la visibilidad del arte rupestre (más visible, durante más tiempo), 
como herramienta de señalización y/o demarcación de recursos críticos. Este posible uso del arte como marcador del espacio y los recursos será explorado más detalladamente en futuras investigaciones.

En términos de variabilidad morfológica de las imágenes rupestres, evaluada a escala de presencia/ ausencia de clases, no se observan diferencias significativas: todas las áreas biogeográficas exhiben repertorios de clases similares, lo cual sugiere la existencia de un fenómeno de circulación de información visual regional ${ }^{3}$. Sin embargo, al analizar las frecuencias de clases, los análisis de agrupamiento jerárquico indican que Patagonia y Monte presentan mayores similitudes entre sí que con el área Altoandina y que, a su vez, los sitios de Monte y Patagonia ubicados en La Payunia son más afines con sitios de otros sectores de Patagonia que con sitios de otros sectores de Monte. Estos resultados son consistentes con la historia de ocupación humana del sur de Mendoza a escala regional, pero marcan simultáneamente tendencias específicas a escala intrarregional. Así, habiendo partido de una premisa según la cual la producción de arte rupestre acompañó todo el proceso de poblamiento humano de la región, sería esperable que los sectores más productivos de Monte y Patagonia (no pertenecientes a La Payunia) tuvieran más elementos compartidos entre sí que con los sectores menos productivos como La Payunia y Altoandina, dado que registran historias de poblamiento más largas y una ventana de tiempo mayor para el desarrollo de interacciones. Sin embargo, los sectores más productivos de Patagonia, tales como los valles intermontanos y el piedemonte, comparten más similitudes de repertorio y frecuencias de clases de motivos con el sector menos productivo de La Payunia, la cual posee una historia de ocupación más reciente (pos 2000 años AP), tanto en Patagonia como en Monte. Este resultado permite aportar dos importantes conclusiones preliminares: por un lado, las tendencias de distribución espacial de las clases de motivos son consistentes con las estimaciones cronológicas tardías para el arte rupestre de la región y, por otro lado, dichas tendencias vinculan a la producción rupestre de La Payunia con un potencial origen principalmente patagónico.

En este contexto, si las diferencias observadas entre las figuras cefalomorfas-mascariformes (distribuidas principalmente entre el área de Patagonia y el sector de La Payunia correspondiente al área de Monte) y los motivos geométricos complejos rectilíneos pintados (distribuidos principalmente por el área de Monte) fueran manifestaciones relativamente contemporáneas ${ }^{4}$, podrían ser indicativas del surgimiento de un escenario de diferenciación entre áreas biogeográficas hacia finales del Holoceno tardío, en un contexto de intensificación y competencia por los recursos. Sin embargo, estas diferencias habrían funcionado como marcas visuales específicas dentro de un repertorio principalmente signado por la similitud general de clases de motivos, lo que sugiere que el proceso más preponderante habría sido la circulación de información arriba mencionada.

En síntesis, la visión regional del arte rupestre del sur de Mendoza permite destacar que su producción se caracteriza por un repertorio de diez clases de motivos distribuidas en las tres áreas biogeográficas bajo estudio, las que en su mayoría se combinan entre sí en los sitios analizados. Esta distribución y combinación de clases de motivos sugiere la existencia de amplios procesos de comunicación visual relativamente homogéneos (clases de motivos compartidas y combinadas), pero puntuados por tendencias específicas en la no-combinación de ciertas clases (cefalomorfas-mascariformes vs. geométricos complejos rectilíneos), cada una producida principalmente por técnicas específicas (grabado vs. pintura) y ubicada en determinados emplazamientos (sin reparo vs. con reparo). De esta manera, se comienzan a vislumbrar procesos de producción artística y comunicación visual que habrían operado en distintas escalas espaciales - iy temporales?- cuya pesquisa requerirá de indagar en el futuro sobre datos de mayor resolución morfológica, técnica y contextual.

\section{Agradecimientos}

Agradecemos a las familias de la zona rural por recibirnos en sus casas y brindarnos su conocimiento, al equipo técnico del CRIDC-MRM y a los/las evaluadores por su valiosas correcciones y sugerencias. El trabajo del Dr. Agustín Acevedo se desarroIla en el marco de una Beca Interna Posdoctoral CONICET.

\section{REFERENCIAS CITADAS}

Aschero, C. (1997). De cómo interactúan emplazamientos, conjuntos y temas. Revista del Museo de Historia Natural de San Rafael, 16(1/4), 17-28. 
Borrero, L. A. (1994-1995). Arqueología de la Patagonia. Palimpsesto, 4, 9-69.

Cabrera, A. (1976). Regiones fitogeográficas argentinas. En W. F. Kugler (Ed.), Enciclopedia Argentina de Agricultura y Jardinería (pp. 1-85). Acme.

Carden, N. (2009). Imágenes a través del tiempo. Arte rupestre y construcción social del paisaje en la Meseta Central de Santa Cruz. Sociedad Argentina de Antropología.

Conkey, M. (1984). To find ourselves: art and social geography of prehistoric hunter-gatherers. En C. Shire (Ed.), Past and present in Hunter-Gatherer studies (pp. 253-276). Academic Press.

Dieguez, S. y Neme, G. A. (2003). Geochronology of the archaeological site Arroyo Malo 3 and the first human occupations in the North Patagonia early Holocene. En L. Miotti, M. Salemme, y N. Flegenheimer (Eds.), Ancient Evidence for Paleo South Americans: from where the south winds blows (pp. 87-92). Center for the Study of the First Americans, Texas A\&M University Press.

Gil, A. F. (1997-1998). Cultígenos prehispánicos en el sur de Mendoza. Discusiones en torno al límite meridional de la agricultura andina. Relaciones de la Sociedad Argentina de Antropología, XXII-XXIII, 295-313.

Gil, A. F. (2006). Arqueología de La Payunia. BAR International Series 1477, Archaeopress.

Gil, A. F. y Neme, G. A. (2013). Fronteras en el registro arqueológico: preguntas, casos y debates en el Centro Occidente Argentino. En M. Gascón y M. J. Ots (Eds.), Fronteras y periferias en arqueología e historia (pp. 1130). Dunken.

Gil, A. F, Neme, G. A. y Tykot, R. (2010). Isótopos estables y consumo de maíz en el Centro Occidente Argentino: tendencias temporales y espaciales. Chungara, 42(2), 497-513.

Gil, A. F., Zárate, M. y Neme, G. A. (2006). Mid-Holocene paleoenvironments and the archaeological record of southern Mendoza, Argentina. Quaternary International, 132, 81-94.

Gradin, C. (1997-1998). El arte rupestre del sur mendocino entre los siglos VIII y XV de la era. ¿Un área de conflicto o de convivencia? Relaciones de la Sociedad Argentina de Antropología, XXII-XXIII, 7-23.

Hart, L. (2015). Arte de la prehistoria. Diseños rupestres de Cuyo. Zeta Editores.

Hernández Llosas, M. I. (1997). Aportes de los estudios de arte rupestre al avance del conocimiento en Arqueología. Revista del Museo de Historia Natural de San Rafael, 16(1/4), 29-40.
Lagiglia, H. A. (1956a). La Gruta del Indio del Rincón del Atuel. Un reparo con pinturas rupestres de San Rafael (Mendoza). Revista Científica de Investigaciones del Museo de Historia Natural de San Rafael, 1(1), 5-18.

Lagiglia, H. A. (1956b). Estudios Arqueológicos en el Rincón del Atuel (Dpto. San Rafael, Mendoza). Anales de Arqueología y Etnología, 12, 229-288.

Lagiglia, H. A. (1962-1968). Secuencias culturales del Centro-Oeste Argentino: valles del Atuel y Diamante. Revista Científica de Investigaciones del Museo de Historia Natural de San Rafael, 1(4), 159-174.

Lagiglia, H. A. (1977). Arqueología y Ambiente Natural de los Valles del Atuel y Diamante [Tesis doctoral inédita. Universidad Nacional de La Plata].

Lagiglia, H. A. (1997). Estudio de los petroglifos de Ponontrehue (sur de Mendoza). Revista del Museo de Historia Natural de San Rafael, 16(1/4), 269-287.

Lagiglia, H. A. (1980). El proceso de agriculturización del Sur de Cuyo: La Cultura del Atuel II. En Actas del V Congreso Nacional de Arqueología Argentina (pp. 231 252). Universidad Nacional de San Juan.

Lagiglia, H. A. (2003). Arqueología y arte rupestre de Las Tinajas del sur de Mendoza. Museo de Historia Natural de San Rafael; Municipalidad de San Rafael.

Lagiglia, H. A. (2004). La posible significación mágica del arte rupestre en el Atuel. Notas del Museo de Historia Natural de San Rafael, 55, 5-21.

Martel, A. (2006). Arte rupestre y espacios productivos en el formativo: Antofagasta de la Sierra (Puna meridional, Argentina). En D. Fiore y M. M. Podestá (Eds.), Tramas en la Piedra. Producción y usos del Arte Rupestre (pp. 157-167). World Archaeological Congress; Amigos del Instituto Nacional de Antropología.

Neme, G. A. (2007). Cazadores-recolectores de altura en los Andes meridionales: el alto valle del río Atuel. BAR International Series 1591, Archaeopress.

Neme, G. A. y Gil, A. F. (2008). Biogeografía Humana en los Andes Meridionales: tendencias arqueológicas en el Sur de Mendoza. Chungara, 40(1), 5-18.

Neme, G. A. y Gil, A. F. (2009). Human occupation and increasing mid-Holocene aridity. Current Anthropology, 50, 149-163.

Neme, G. A. y Gil, A. F. (2012). El registro arqueológico del sur de Mendoza en perspectiva biogeográfica. En G. A. Neme y A. F. Gil (Eds.), Paleoecología humana en el sur de Mendoza: perspectivas arqueológicas (pp. 255-279). Sociedad Argentina de Antropología.

Neme, G. A., Gil, A. F., y Durán, V. (2005). Late Holocene in southern Mendoza (northwestern Patagonia): radiocarbon pattern and human occupation. Before Farming, 2(artículo 5), 1-18. 
Neme, G. A., Gil, A. F., Garvey, R., Llano, C. L., Zangrando, A. F., Francheti, F., De Francesco, C. G. y Michieli, C. T. (2011). El registro arqueológico de la Gruta de El Manzano y sus implicancias para la arqueología de Nordpatagonia. Magallania, 9, 243-265.

Otaola, C., Wolverton, S., Giardina, M. y Neme, G. A. (2015). Geographic scale and zooarchaeological analysis of late Holocene foraging adaptations in western Argentina. Journal of Archaeological Science, 55, 16-25.

Romero, G. y Re, A. (2014). Representaciones rupestres del noreste de Neuquén (Patagonia Septentrional). Primeras tendencias espaciales y temporales. Comechingonia, 18, 73-92.

Rusconi, C. (1962). Poblaciones pre y posthispánicas de Mendoza, vol. III. Gobierno de Mendoza.

Scheinsohn, V. (2011). Rock Art Information among HunterGatherers in Northwest Patagonia: An Assessment of Broad Scale and Territorial Models. En R. Whallon, W. Lovis y R. Hitchcock (Eds.), Information and its Role in Hunter-Gatherer Bands (pp. 235-247). Cotsen Institute of Archaeology Press; University of California Press.

Schobinger, J. (1978). Nuevos lugares con arte rupestre en el extremo sur de la provincia de Mendoza. Relaciones de la Sociedad Argentina de Antropología, XII, 175-182.

Schobinger, J. (2002). Arte Rupestre del Departamento Malargüe. En A. F. Gil y G. A. Neme (Eds.), Entre Montañas y Desiertos: Arqueología del Sur de Mendoza (pp. 181-194). Sociedad Argentina de Antropología.

Semper, J. V. y Lagiglia, H. A. (1968). Excavaciones arqueológicas en el Rincón del Atuel. Revista Científica de Investigaciones del Museo de Historia Natural de San Rafael, 1(4), 89-158.
Tucker, H., Risi, A. y Bandiera, R. (2011). Identification of astronomical objects in ancient engravings: Malargüe, Mendoza, Argentina. Methodological contributions in archaeoastronomy. Proceedings of the International Astronomical Union, 7(278), 118-127.

Veth, P. (1989). Islands in the interior: a model for the colonization of Australia's arid zone. Archaeology in Oceania, 24, 81-92.

Wheatley, D. y Gillings, M. (2002). Spatial Technology and Archaeology. Taylor \& Francis.

\section{NOTAS}

1.- Se calcularon además los índices de sitios con arte por $\mathrm{km}^{2}$ para cada área biogeográfica: estos confirman que Patagonia registra más sitios que las áreas Altoandina y Monte (Tabla 1). Sin embargo, cabe aclarar que estos índices se calcularon sobre las superficies totales de las áreas, sin tomar en cuenta variaciones altitudinales, de pendientes, ni variaciones de disponibilidad en los soportes rocosos, factores que sin dudas han influido sobre la distribución de sitios y que serán evaluados en el futuro.

2.- Es frecuente encontrar que un mismo sitio es denominado de distintas maneras por autores diferentes, por ejemplo: alero, cueva, reparo, abrigo, etcétera.

3.- No se descarta la presencia de sesgos asociados al tamaño de la muestra analizada $(n=61$ de 96$)$ o a la baja resolución de la escala de "clases" usada para la clasificación morfológica de motivos.

4.- De acuerdo con las estimaciones relativas realizadas por investigadores antecedentes, las figuras cefalomorfas-mascariformes se ubicarían temporalmente en ca. 1300 años AP (Gradin, 1997-1998), y los geométricos complejos rectilíneos pintados, en ca. 1400-500 años AP (Lagiglia, 1977, 2003; Gradin, 1997-1998; Schobinger, 2002). 
